\title{
Afferents to the Ventrolateral Preoptic Nucleus
}

\author{
Thomas C. Chou, ${ }^{1,2}$ Alvhild A. Bjorkum, ${ }^{1,3}$ Stephanie E. Gaus, ${ }^{1,2}$ Jun Lu, ${ }^{1}$ Thomas E. Scammell, ${ }^{1}$ and \\ Clifford B. Saper ${ }^{1,2}$ \\ ${ }^{1}$ Department of Neurology and 2Program in Neuroscience, Harvard Medical School, Beth Israel Deaconess Medical \\ Center, Boston, Massachusetts 02215, and '3epartment of Physiology, University of Bergen, Bergen N-5009, Norway
}

\begin{abstract}
Sleep is influenced by diverse factors such as circadian time, affective states, ambient temperature, pain, etc., but pathways mediating these influences are unknown. To identify pathways that may influence sleep, we examined afferents to the ventrolateral preoptic nucleus (VLPO), an area critically implicated in promoting sleep. Injections of the retrograde tracer cholera toxin B subunit (CTB) into the VLPO produced modest numbers of CTB-labeled monoaminergic neurons in the tuberomammillary nucleus, raphe nuclei, and ventrolateral medulla, as well as a few neurons in the locus coeruleus. Immunohistochemistry for monoaminergic markers showed dense innervation of the VLPO by histaminergic, noradrenergic, and serotonergic fibers. Along with previous findings, these results suggest that the VLPO and monoaminergic nuclei may be reciprocally connected. Retrograde and anterograde tracing showed moderate or heavy inputs to the VLPO from hypothalamic regions including the median preoptic nucleus, lateral hypothalamic area, and
\end{abstract}

dorsomedial hypothalamic nucleus (DMH), autonomic regions including the infralimbic cortex and parabrachial nucleus, and limbic regions including the lateral septal nucleus and ventral subiculum. Light to moderate inputs arose from orexin and melanin concentrating hormone neurons, but cholinergic or dopaminergic inputs were extremely sparse. Suprachiasmatic nucleus (SCN) projections to the VLPO were sparse, but the heavy input to the VLPO from the $\mathrm{DMH}$, which receives direct and indirect SCN inputs, could provide an alternate pathway regulating the circadian timing of sleep. These robust pathways suggest candidate mechanisms by which sleep may be influenced by brain systems regulating arousal, autonomic, limbic, and circadian functions.

Key words: VLPO; afferents; autonomic; limbic; circadian; ventrolateral; preoptic; sleep; $\mathrm{DMH}$; dorsomedial; hypothalamus; histamine; noradrenaline; serotonin; orexin
Recent studies have identified galaninergic, GABAergic neurons in the ventrolateral preoptic nucleus (VLPO) that are sleep active and may promote sleep (Sherin et al., 1998). VLPO neurons express the immediate-early gene product Fos, a possible marker of neuronal activity, in proportion to time spent asleep (Sherin et al., 1996; Gaus and Saper, 1999; Lu et al., 2000b). The firing rates of VLPO neurons also increase during sleep, particularly during deeper stages of sleep when electroencephalographic (EEG) slow waves are most intense (Szymusiak et al., 1998). VLPO lesions produce long-lasting reductions of sleep time and EEG slow wave amplitude, suggesting that VLPO activity may promote, maintain, or intensify sleep (Lu et al., 2000a). These effects on sleep may be mediated by inhibitory projections from the VLPO to wake-active monoaminergic nuclei such as the tuberomammillary nucleus (TMN), raphe nuclei, and locus coeruleus (Sherin et al., 1996, 1998; Steininger et al., 2001). Somnogens such as adenosine agonists or prostaglandin D2 increase Fos expression in VLPO neurons and promote sleep, particularly when injected near the VLPO (Matsumura et al., 1994; Scammell et al., 1998a; Scammell et al., 2001).

Identifying afferents to the VLPO may help explain how sleep

Received April 20, 2001; revised Aug. 29, 2001; accepted Oct. 25, 2001.

This work was supported by United States Public Health Service Grants NS33987, HL60292, and MH01507. We thank Quan Ha for excellent technical support, and Sonsoles de Lacalle for ChAT immunostained tissue.

Correspondence should be addressed to Dr. Clifford B. Saper, Department of Neurology, Beth Israel Deaconess Medical Center, 330 Brookline Avenue, Boston, MA 02215. E-mail: csaper@caregroup.harvard.edu

Copyright (C) 2002 Society for Neuroscience $\quad 0270-6474 / 02 / 220977-14 \$ 15.00 / 0$ is influenced by diverse factors including light, circadian time, ambient temperature, affective states, and pain (Sakaguchi et al., 1979; Eastman et al., 1984; Alfoldi et al., 1991; McGinty et al., 1994; Roehrs et al., 1994; Dijk and Czeisler, 1995). However, only a few afferents to the VLPO are known, including a direct input from the retina ( $\mathrm{Lu}$ et al., 1999) and a sparse input from the suprachiasmatic nucleus (SCN) (Gaus and Saper, 1998; Novak and Nunez, 2000). To determine systematically other inputs to the VLPO, we injected a retrograde tracer into the VLPO and placed anterograde tracers into regions identified by retrograde tracing. We also used immunostaining for monoaminergic markers to further characterize monoaminergic inputs to the VLPO.

\section{MATERIALS AND METHODS}

Animals. All experiments used male Sprague Dawley rats weighing 275-350 gm. All work was approved by Harvard Medical School and Beth Israel Deaconess Medical Center Animal Care and Use committees.

Retrograde and anterograde tracing. Rats were anesthetized with chloral hydrate $(350 \mathrm{mg} / \mathrm{kg}$, i.p.) and placed in a stereotaxic apparatus. Tracers were injected via a glass pipette (tip diameter 10-20 $\mu \mathrm{m}$ ) using a compressed air delivery system as described previously (Elmquist and Saper, 1996; Scammell et al., 1998b). Injections used the retrograde tracer cholera toxin B subunit (CTB) (List Biological Labs, Campbell, $\mathrm{CA} ; 1 \%$ in saline; $1-3 \mathrm{nl})$ or the anterograde tracers biotinylated dextranamine (BD) (Molecular Probes, Eugene OR; 25\% in saline; 1-20 nl), Phaseolous vulgaris leucoagglutinin (PHAL) (Vector Labs, Burlingame, CA; $2.5 \%$ in PBS, $\mathrm{pH} 8.0$ ), or wheat germ agglutinin-horseradish peroxidase (WGA-HRP; Sigma, St. Louis, MO; $1 \%$ in PBS). PHAL was iontophoresed using $4 \mu \mathrm{A}$ positive current, $7 \mathrm{sec}$ on $/ 7 \mathrm{sec}$ off, for $15 \mathrm{~min}$. VLPO coordinates (measured with skull leveled) were $-0.4 \mathrm{~mm}$ rostral to bregma, $-8.4 \mathrm{~mm}$ ventral to the dura, and $-0.9 \mathrm{~mm}$ lateral to the midline. Coordinates for other regions were adapted from the atlas of 


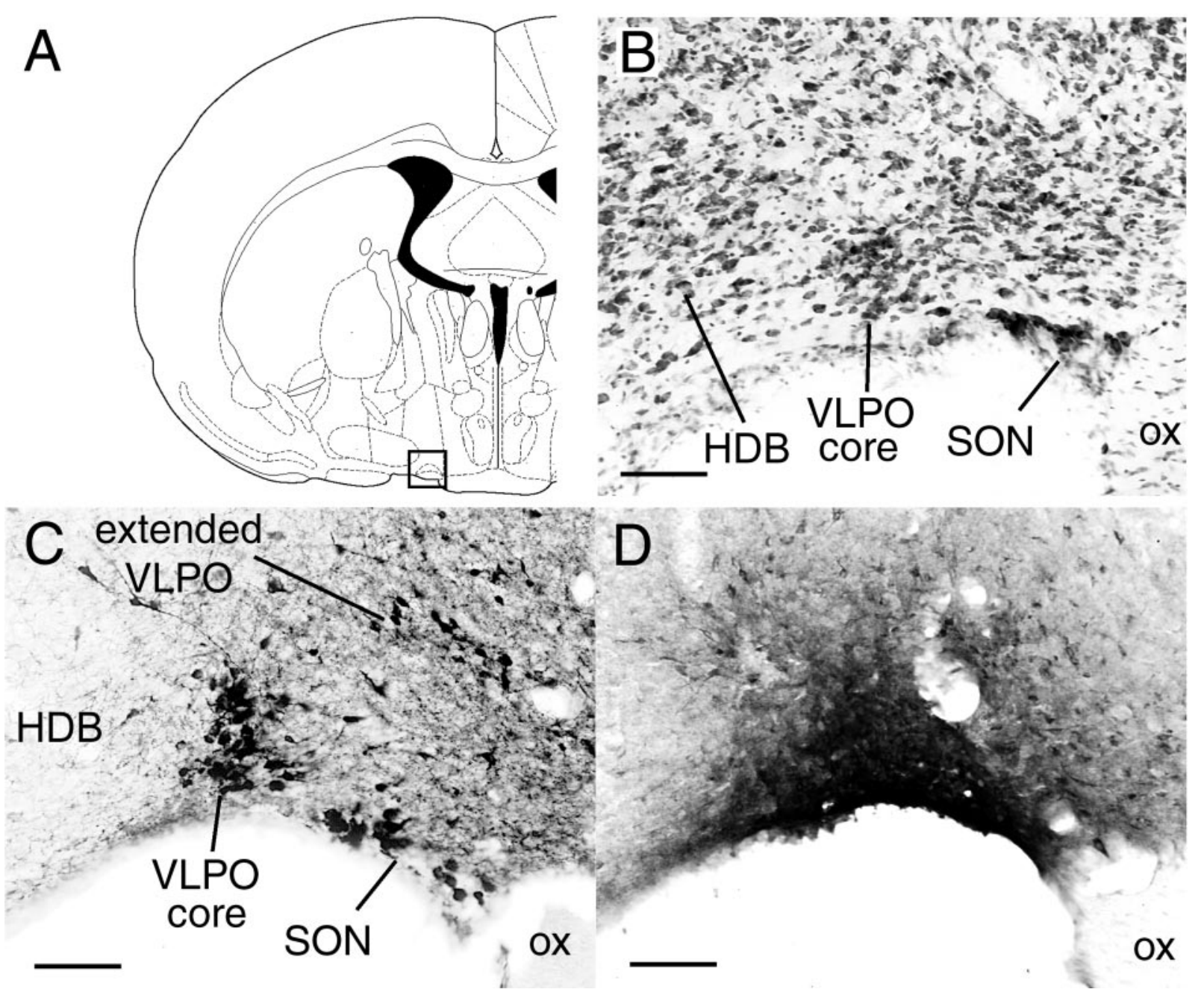

Figure 1. A, Coronal atlas section (Paxinos and Watson, 1997) with square outline showing lateral preoptic region enlarged in $B-D$. $B$, Nissl-stained section shows cell-dense VLPO core in relation to adjacent structures such as the horizontal nucleus of the diagonal band (HDB) and supraoptic nucleus $(S O N)$. C, Galanin immunostaining shows a dense cluster of immunoreactive neurons in the VLPO core and diffusely distributed neurons in the extended VLPO, which is dorsal and medial to the VLPO core. D, CTB injection into VLPO core (case 40 ). Scale bars, $100 \mu \mathrm{m}$.

Paxinos and Watson (1997). After 7-14 d, animals were deeply anesthetized with chloral hydrate and transcardially perfused with $4 \%$ paraformaldehyde. Some animals received $12 \mu \mathrm{l}$ of $1.5 \%$ colchicine into the lateral ventricle $24-48 \mathrm{hr}$ before they were killed to enhance galanin immunoreactivity in cell bodies.

Tissue preparation. After perfusion, brains were removed, post-fixed in $4 \%$ paraformaldehyde for 1-2 hr, and equilibrated overnight in PBS with $20 \%$ sucrose and $0.01 \%$ sodium azide. Brains stained for histamine were perfused with a mixture of $4 \%$ paraformaldehyde and $4 \%$ 1-ethyl-3-(3dimethylamino-propyl) carbodiimide. Brains were cut on a freezing microtome into five series of $30 \mu \mathrm{m}$ sections, which were stored at $4^{\circ} \mathrm{C}$ in PBS with $0.01 \%$ sodium azide.

Immunohistochemistry. Endogenous peroxidases were inactivated by a 30 min incubation in PBS with $1 \%$ hydrogen peroxide, followed by three rinses of $5 \mathrm{~min}$ each in PBS. Tissue was incubated overnight at room temperature in one of the following primary antisera: goat anti-CTB (List Biological Labs) at 1:100,000 dilution; goat anti-PHAL (Vector Labs) at 1:10,000; rabbit anti-galanin (Peninsula Labs) at 1:10,000; sheep anti-adenosine deaminase (ADA) (gift from R. Kellems, University of Texas, Houston, TX) at 1:25,000; rabbit anti-dopamine $\beta$-hydroxylase $(\mathrm{DBH})$ (Eugene Tech) at 1:10,000; rabbit anti-tyrosine hydroxylase (TH) (Eugene Tech) at 1:500; guinea pig anti-serotonin transporter (5HTT) (Chemicon) at 1:5000; rabbit anti-serotonin (5HT) (Incstar) at 1:10,000; rabbit anti-dopamine transporter (DAT; gift from R. Vaughan, University of North Dakota) at 1:10,000; rabbit anti-orexin (gift from M. Yanagisawa, University of Texas, Southwestern Medical Center) at
1:10,000; rabbit anti-choline acetyltransferase (ChAT) (gift from L. Hersh, University of Kentucky) at 1:10,000; or rabbit anti-histamine at 1:1000 (gift from P. Panula, Abo Akademi University, Turku, Finland). Primary antisera were diluted in PBS with $0.25 \%$ Triton X-100 (PBT) and $0.01 \%$ sodium azide. After primary incubation, tissue was rinsed three times for $5 \mathrm{~min}$ in PBS, incubated in biotinylated secondary antibody (donkey anti-guinea pig IgG, donkey anti-goat IgG, donkey anti-sheep IgG, or donkey anti-rabbit IgG, 1:1000; Jackson ImmunoResearch, West Grove, PA) for $1 \mathrm{hr}$, rinsed three times for 5 min in PBS, incubated in avidin-biotin complex (ABC) (Vector) for $1 \mathrm{hr}$, then rinsed three times for $1 \mathrm{~min}$. Reaction product was visualized by incubation in $1 \%$ diaminobenzidine (DAB) with $0.01 \%$ hydrogen peroxide for $1-10$ min. Tissue was rinsed in PBS-azide and mounted on gelatin-coated glass slides. In some cases, $0.05 \%$ nickel ammonium sulfate and $0.05 \%$ cobalt chloride were added to the DAB solution to darken the reaction product. Staining for biotinylated dextrans (BDs) required only incubation in the $\mathrm{ABC}$ complex and DAB as described above. Some cases were double stained first for BD using nickel and cobalt enhancement, followed by immunostaining for galanin without nickel or cobalt, yielding blue-black BD fibers and brown galanin-immunoreactive cells.

WGA-HRP-injected tissue was processed using a tetramethyl benzidine method described previously (Mesulam, 1978).

In some cases, histochemical detection of BD was enhanced using a tyramide signal amplification kit (TSA-biotin; NEN, Boston, MA). Briefly, tissue was incubated in $\mathrm{ABC}$ for $1 \mathrm{hr}$, rinsed in PBS, then incubated $10 \mathrm{~min}$ in biotinylated tyramide according to manufacturer's 

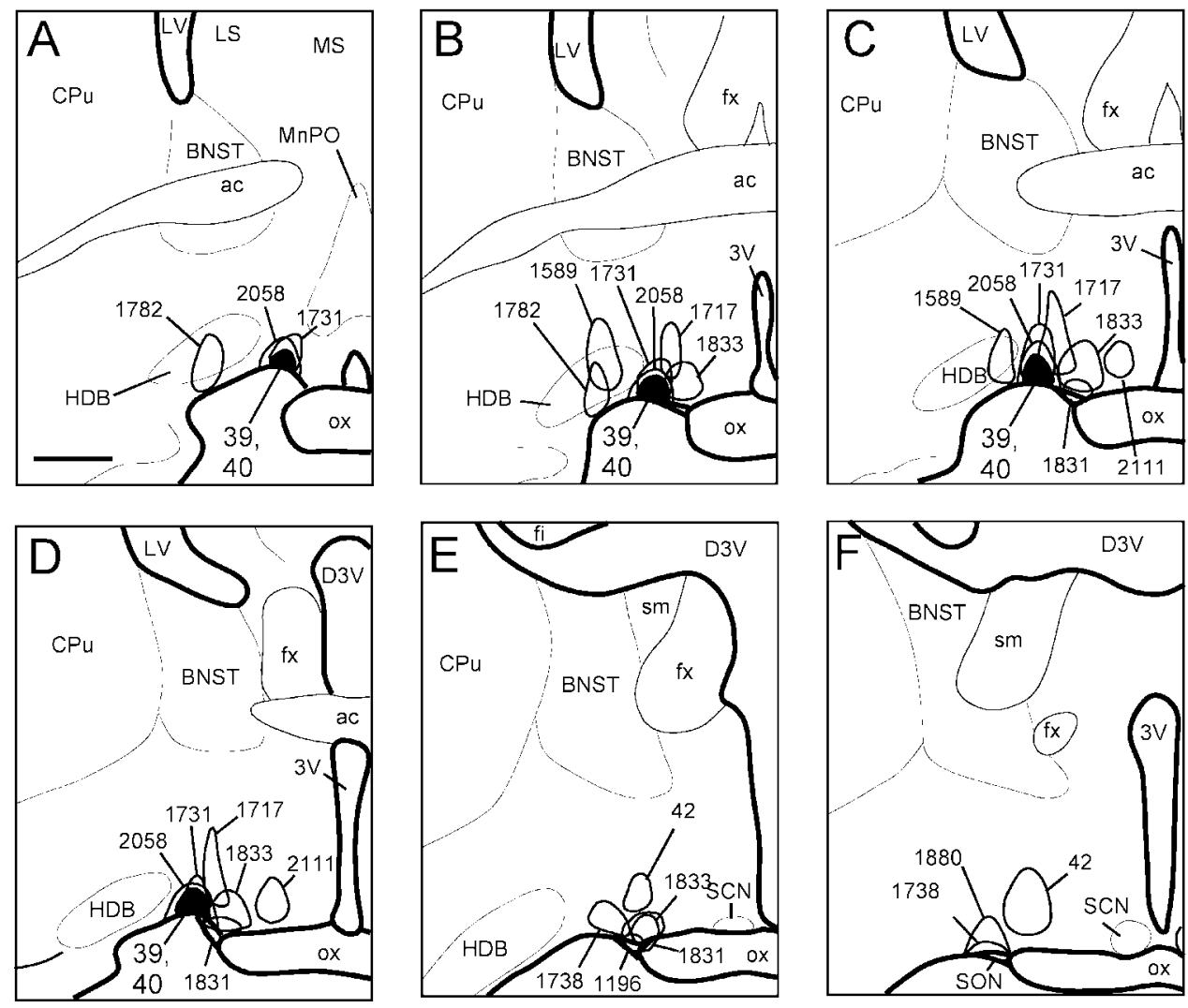

Figure 2. $A-F$, Camera lucida drawings of coronal brain sections outlining representative injection sites into the VLPO and adjacent areas. Drawings are ordered from most rostral to most caudal. The filled outline indicates cases 39 and 40, which show the two injection sites most confined to the VLPO core. Scale bar, $500 \mu \mathrm{m}$. directions. The deposited tyramide was detected by another $1 \mathrm{hr}$ incubation in ABC complex, followed by DAB detection.

Sections were mounted on gelatin-coated glass slides, dehydrated in graded alcohols, and delipidated in xylenes. Slides were Nissl counterstained for 1-5 min at room temperature in either Giemsa solution [0.1\% Giemsa (Sigma) in $6.2 \%$ methanol, $3.8 \%$ glycerin, and $90 \%$ monobasic phosphate buffer, $\mathrm{pH} 4.5$ ] or thionin $(0.25 \%$ in $0.2 \mathrm{M}$ acetate buffer, $\mathrm{pH}$ 4.5). Slides were coverslipped using Permaslip (Alban Scientific Inc.) mounting medium.

Fluorescent double labeling. We used fluorescence immunostaining to colocalize CTB with monoaminergic markers, as well as markers for cholinergic and orexinergic cells. Tissue was incubated overnight at room temperature in PBT with $0.01 \%$ sodium azide containing goat anti-CTB (1:30,000, List Biological) and with one of the following: rabbit anti-TH (1:250); rabbit-anti-5HT (1:1000); rabbit anti-ChAT $(1: 10,000)$; rabbit anti-orexin $(1: 10,000)$, or sheep anti-ADA $(1: 25,000)$ for $2 \mathrm{~d}$ at room temperature. Tissue was rinsed in PBS, incubated $1 \mathrm{hr}$ in a mixture of biotinylated donkey anti-goat and $\mathrm{Cy} 3$-conjugated donkey anti-rabbit secondary antibodies (both at 1:1000; Jackson ImmunoResearch), and then $1 \mathrm{hr}$ in Alexafluor-488 streptavidin (1:1000, Molecular Probes). Because the ADA primary antiserum was raised in sheep and is antigenically similar to goat immunoglobulins, we replaced the goat antiCTB primary with a rabbit-anti-CTB primary (Sigma) at 1:10,000 dilution to avoid cross-reactivity. Immunohistochemical controls were performed in which one or both primary antibodies were omitted. These controls showed no double-labeled cells.

Silver intensification. In some cases, BD staining was enhanced using a silver intensification method described previously (Kitt, 1988). Briefly, we incubated slides at $55^{\circ} \mathrm{C}$ for $45 \mathrm{~min}$ in a saturated aqueous solution of $1 \%$ silver nitrate neutralized with ammonium hydroxide, rinsed for 15 min in room temperature water, and then incubated 15 min in $0.1 \%$ gold chloride solution at room temperature. The silver-gold complex was stabilized in a $5 \%$ sodium thiosulfate solution for $5 \mathrm{~min}$.

Anatomic identification of VLPO core and extended VLPO. The VLPO nucleus was initially defined by retrograde labeling from the TMN, which showed a distinct cluster of labeled neurons in the lateral preoptic area, roughly half of which expressed Fos protein during sleep but not waking (Sherin et al., 1996). Furthermore, $>80 \%$ of lateral preoptic neurons retrogradely labeled from the TMN expressed galanin (Sherin et al.,
1998), which is a useful marker for this cell group because it is not expressed by adjacent preoptic nuclei except for the supraoptic nucleus (SON).

Recent studies confirmed that $>80 \%$ of VLPO area neurons that express Fos during sleep are galaninergic, and conversely $\sim 50 \%$ of galaninergic neurons in this area express Fos during sleep (Gaus and Saper, 1999; Lu et al., 2000b). In colchicine-treated brains, we therefore identified VLPO neurons using galanin immunohistochemistry (see Fig. $1 C)$. The distribution of galanin reveals two subregions, termed the VLPO "core" and the "extended" VLPO. Both subgroups express Fos protein during sleep and project to the TMN, suggesting that these two regions are related functionally, anatomically, and chemically ( $\mathrm{Lu}$ et al., 2000b). The VLPO core contains the highest density of sleep-active neurons, whereas extended VLPO galanin neurons are diffusely scattered up to $400 \mu \mathrm{m}$ dorsal and medial to the VLPO core (Gaus and Saper, 1999; Lu et al., 2000b). Extended VLPO neurons intermingle with many nongalaninergic neurons that may not be sleep active.

Nissl counterstaining was also used to demarcate the VLPO core (see Fig. $1 B$ ) relative to other anatomic landmarks. On a Nissl stain, the VLPO core is a discrete 100- to $300-\mu \mathrm{m}$-diameter cluster of neurons along the ventral edge of the lateral preoptic area, just lateral to the optic chiasm and just medial to the horizontal limb of the diagonal band nucleus. The most caudal extent of the VLPO core overlaps the coronal plane as the most rostral extent of the SCN, but the VLPO is $\sim 1 \mathrm{~mm}$ lateral to the SCN. Extended VLPO neurons cannot be distinguished on a Nissl stain.

Analysis. Drawings of retrograde or anterograde labeling were made using a camera lucida (Leitz) and digitized, and the lines were redrawn using Canvas software (Deneba Systems, Miami, FL). Photomicrographs were taken with a color digital camera (Kodak DCS460). Images were adjusted for contrast, brightness, and sharpness using Photoshop (Adobe Systems). Retrogradely labeled neuronal profiles were counted using a light microscope and a $25 \times$ objective (Zeiss), whereas anterogradely labeled varicosities were counted using a $100 \times$ oil immersion objective. Because of variability of injection sites and staining, cell and varicosity counts provide only a rough, relative indication of the intensity of projections. Therefore, counts were not corrected for stereological errors caused by cell and terminal diameter. 


\begin{tabular}{|c|c|}
\hline $3 \mathrm{~V}$ & Third ventricle \\
\hline A1 & A1 noradrenergic cells \\
\hline $\mathrm{ac}$ & Anterior commissure \\
\hline $\mathrm{AHN}$ & Anterior hypothalamic nucleus \\
\hline AP & Area postrema \\
\hline $\mathrm{Aq}$ & Cerebral aqueduct \\
\hline BLA & Basolateral amygdaloid nucleus \\
\hline BMA & Basomedial amygdaloid nucleus \\
\hline $\mathrm{C} 1$ & $\mathrm{C} 1$ adrenergic cells \\
\hline $\mathrm{CeA}$ & Central amygdaloid nucleus \\
\hline $\mathrm{CL}$ & Claustrum \\
\hline $\mathrm{Cpu}$ & Caudate-putamen \\
\hline DB & Diagonal band nucleus \\
\hline $\mathrm{DBH}$ & Dopamine $\beta$-hydroxylase \\
\hline DMH & Dorsomedial hypothalamic nucleus \\
\hline DRN & Dorsal nucleus of the raphe \\
\hline fx & Fornix \\
\hline HDB & Horizontal limb of the nucleus of the diagonal band \\
\hline ic & Internal capsule \\
\hline $\mathrm{IC}$ & Inferior colliculus \\
\hline IGL & Intergeniculate leaflet of the thalamus \\
\hline ILC & Infralimbic cortex \\
\hline LA & Lateral anterior hypothalamic nucleus \\
\hline $\mathrm{LC}$ & Locus coeruleus \\
\hline LHA & Lateral hypothalamic area \\
\hline LS & Lateral septal nucleus \\
\hline LV & Lateral ventricle \\
\hline MB & Mammillary bodies \\
\hline MEA & Medial nucleus of the amygdala \\
\hline MGN & Medial geniculate nucleus \\
\hline $\mathrm{MnPO}$ & Median preoptic nucleus \\
\hline MPOA & Medial preoptic nucleus \\
\hline MRN & Median raphe nucleus \\
\hline $\mathrm{mtt}$ & Mammillothalamic tract \\
\hline NAcc & Nucleus accumbens \\
\hline NTS & Nucleus of the solitary tract \\
\hline ot & Optic tract \\
\hline $\mathrm{PBcl}$ & Parabrachial nucleus, central lateral subdivision \\
\hline PR & Perirhinal cortex \\
\hline $\mathrm{PVH}$ & Paraventricular hypothalamic nucleus \\
\hline PVT & Paraventricular nucleus of the thalamus \\
\hline $\mathrm{RMg}$ & Raphe magnus nucleus \\
\hline $\mathrm{SCN}$ & Suprachiasmatic nucleus \\
\hline SFO & Subfornical organ \\
\hline SPZ, SPVH & Subparaventricular zone of the hypothalamus \\
\hline sm & Stria medullaris \\
\hline st & Stria terminalis \\
\hline TMN & Tuberomammillary nucleus \\
\hline $\mathrm{TTd}$ & Tenia tecta, dorsal part \\
\hline VLM & Ventrolateral medulla \\
\hline VLPO & Ventrolateral preoptic nucleus \\
\hline VMH & Ventromedial hypothalamic nucleus \\
\hline VTA & Ventral tegmental area \\
\hline
\end{tabular}

\section{RESULTS}

\section{Experiment 1: CTB injections into the VLPO}

In 100 rats with $\mathrm{CTB}$ injections into the preoptic region, we obtained six cases in which the injection site filled the VLPO core, as identified on a Nissl counterstain. Cases 39, 40, and 1731 were examined in particular detail, because these had the smallest injection sites that spread only slightly to surrounding regions (Figs. $1 D$ and 2 show case 40) (abbreviations in all Figures are defined in Table 1). The injection sites in cases 39 and 40 spread slightly into the rostral corner of the SON (Fig. 1D), whereas the injection site in case 1731 avoided the SON but spread somewhat dorsally to the VLPO core (Fig. 2). The patterns of retrograde labeling in these three cases were very similar and showed that a majority of CTB-labeled neurons were ipsilateral to the injection site, although a few contralateral neurons were seen in a similar distribution (Fig. 3). Larger injections involving the VLPO core confirmed a similar pattern of retrograde labeling but included moderate labeling in additional structures that probably reflected increased spread of the injected tracer to surrounding areas. To control for this spread of tracer, results from VLPO core injections were also compared with control injections placed just outside the VLPO core. Ten such control injections (Fig. 2) are examined and discussed in particular detail.

\section{Hypothalamic afferents}

After VLPO core injections, the largest numbers of CTBlabeled neurons were found in the hypothalamus. Labeled neurons were widely distributed throughout the lateral hypothalamic area (LHA) (Fig. $3 F-I$ ), particularly in regions dorsomedial and ventrolateral to the fornix, and in regions just caudal to the VLPO itself (Fig. $3 E-G$ ). In cases 39, 40, and 1731, LHA neurons accounted for $18 \%$ of all CTB-labeled neurons in the ipsilateral hemisphere (Table 2), more than any other brain region. Many retrogradely labeled neurons were also seen in the median preoptic nucleus (MnPO) (Fig. $3 C$ ), and the DMH (Fig. $3 H, I)$. CTB-labeled cells in these regions represented 9 and $12 \%$ of ipsilateral labeled cells, respectively (Table 2), and labeled neurons were clustered fairly densely, constituting roughly one-fifth of all Nissl-stained neuronal profiles within these two nuclei. In the MnPO, CTB-labeled neurons were dense throughout the nucleus, whereas labeled neurons in the DMH were most dense in caudal and lateral portions of the nucleus ventral to the pars compacta subregion. Retrogradely labeled neurons were rarely seen in the interior of the DMH pars compacta itself (Fig. 3I).

Moderate numbers of labeled neurons were seen in the medial preoptic nucleus (Fig. 3D), anterior hypothalamic nucleus (Fig. $3 E$ ), subparaventricular zone (SPZ) (Fig. 3G), ventromedial hypothalamic nucleus (Fig. $3 H$ ), TMN (Fig. 3J), dorsal hypothalamic area (Fig. 3H), and supramammillary nucleus (Fig. 3J). Each of these regions comprised between 1.5 and $3 \%$ of ipsilateral retrogradely labeled neurons. A few retrogradely labeled neurons were seen in the arcuate nucleus, the subfornical organ, and the dorsomedial SCN. Each of these regions contained $<1.5 \%$ of total retrogradely labeled neurons.

Finally, a moderate number of CTB-labeled neurons were seen immediately dorsal and medial to the injection site itself. These neurons may have been labeled by dendritic uptake of tracer; hence it is difficult to determine the actual connectivity of these neurons with the VLPO.

\section{Telencephalic afferents}

Moderate to high numbers of CTB-labeled neurons were seen in layer $\mathrm{V}$ of the infralimbic cortex (Fig. $3 A$ ), the lateral septal nucleus (Fig. 3B,C), and the ventral subiculum (Fig. $3 K$ ). 

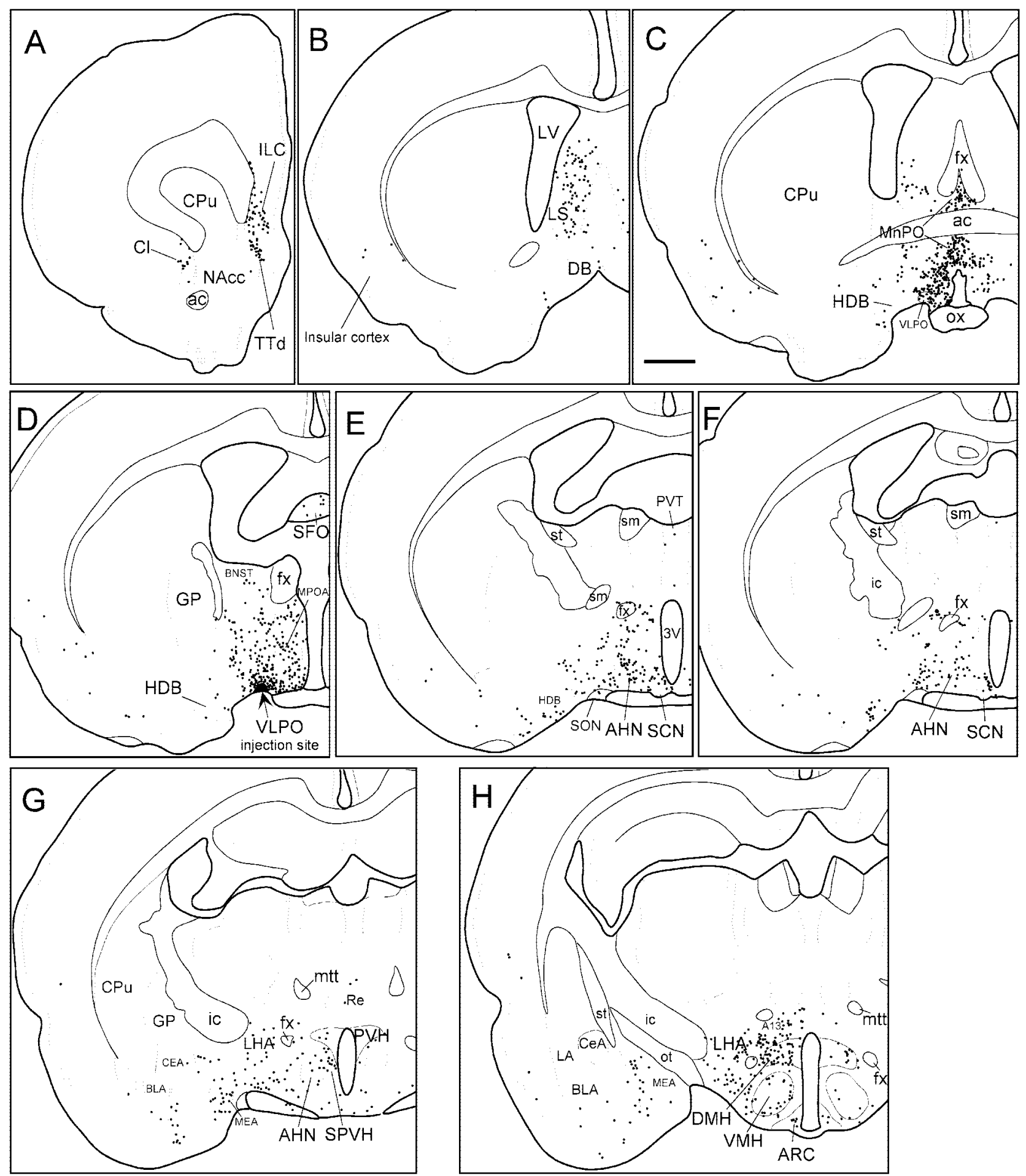

Figure 3. Camera lucida drawings of retrogradely labeled neurons in sections from case 40 . Sections are ordered from most rostral $(A)$ to most caudal $(P)$. Each dot represents one retrogradely labeled neuron. The arrow in $D$ indicates VLPO injection site. Scale bar, 1 mm. (Figure 3 continues.)

These three regions accounted for $\sim 5,15$, and $6 \%$ of ipsilateral CTB-labeled neurons, respectively. Deep layers of the dorsal taenia tecta region, just ventral to the infralimbic cortex, also contained some retrogradely labeled neurons (Fig. $3 A$ ). Low to moderate numbers of neurons were seen in the medial amygdaloid nucleus (Fig. 3G-I), the claustrum (Fig. 3A), and the insular and perirhinal cortex (Fig. $3 B-D)$. Each of these areas contained $2-3 \%$ of ipsilateral retrogradely labeled neurons. Labeled neurons were never seen in the striatum, the hippocampus, or the neocortical regions. CTB-labeled neurons were rarely seen in the cholinergic medial septal and diagonal band nuclei, and the few CTB-labeled neurons in these regions did not have the magnocellular morphology typical of cholinergic neurons. 

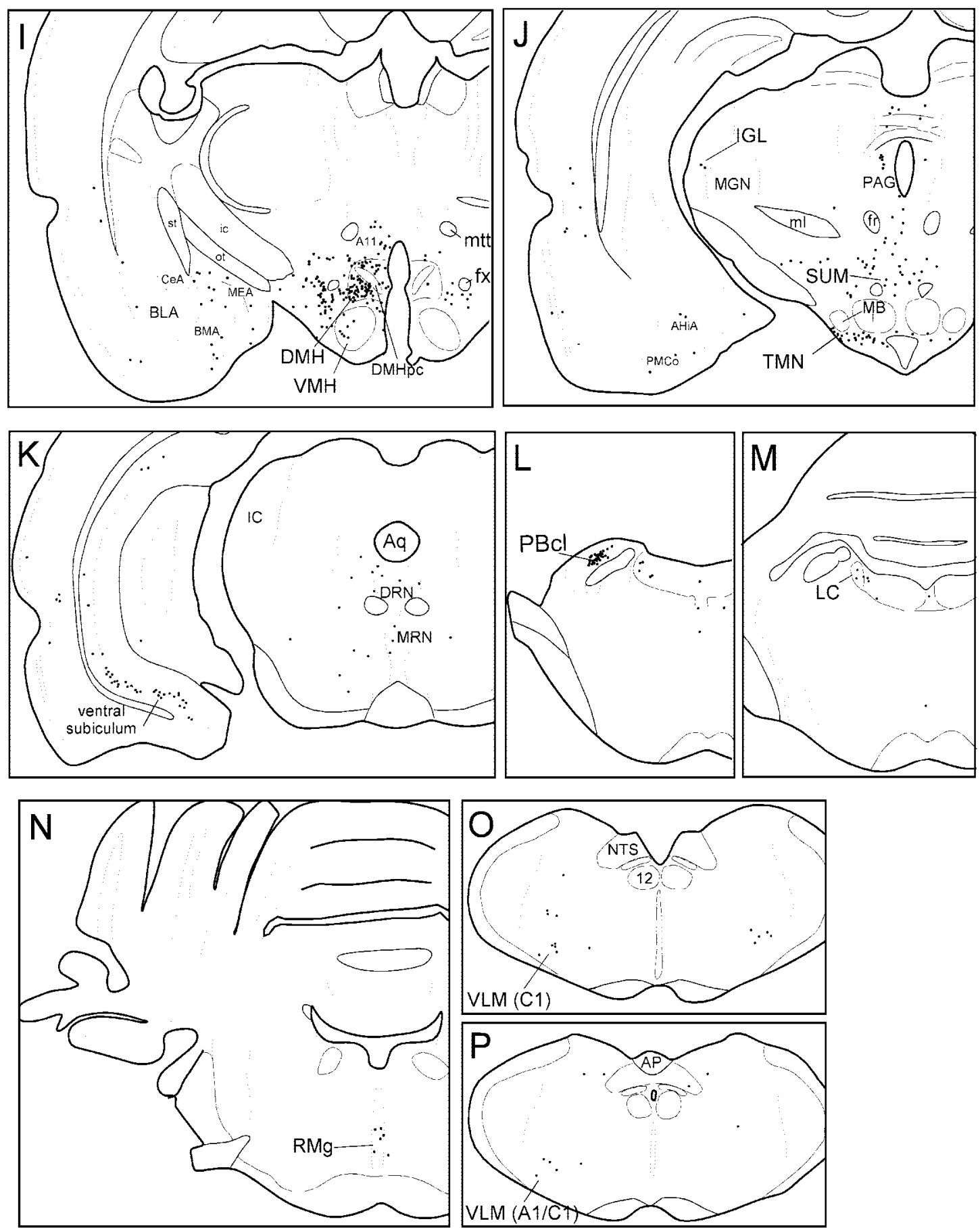

Figure 3 continued.

\section{Thalamic and epithalamic afferents}

A small number of retrogradely labeled neurons (zero to four cells per section) were seen in the paraventricular thalamic nucleus (Fig. $3 F$ ), the reuniens nucleus of the thalamus (Fig. 3G), the lateral habenular nucleus, and the intergeniculate leaflet of the thalamus (Fig. $3 J$ ).

\section{Brainstem afferents}

A dense cluster of retrogradely labeled neurons was seen in the central lateral parabrachial subnucleus and a few neurons extended into the dorsal lateral and external lateral parabrachial subnuclei (Fig. 3L). We found a modest number of CTB-labeled neurons in the dorsal raphe nucleus, particularly in the lateral wings of this nucleus, with fewer neurons in the central linear and median raphe nuclei (Fig. $3 K$ ), and in the nucleus raphe magnus (Fig. $3 N$ ). CTB-labeled neurons were not seen in the more caudal raphe subregions, such as the raphe pallidus or obscurus. Labeled neurons in the raphe were distributed over a large number of brain sections and hence were not prominently clustered in any particular level of the brain. However, the total number of CTBlabeled raphe neurons was comparable to that in the TMN, where 


\begin{tabular}{|c|c|c|}
\hline Region & $\begin{array}{l}\text { CTB-labeled cells } \\
\text { (average } \pm \text { SEM) }\end{array}$ & $\%$ of total \\
\hline Infralimbic cortex & $148 \pm 28$ & $5.3 \pm 0.4$ \\
\hline Claustrum & $59 \pm 3$ & $2.1 \pm 0.4$ \\
\hline Median preoptic nucleus & $263 \pm 51$ & $9.4 \pm 0.4$ \\
\hline Lateral septal nucleus & $396 \pm 71$ & $14.2 \pm 1.8$ \\
\hline Medial preoptic nucleus & $59 \pm 14$ & $2.1 \pm 0.2$ \\
\hline Anterior hypothalamic area & $69 \pm 26$ & $2.5 \pm 0.6$ \\
\hline Suprachiasmatic nucleus & $19 \pm 4$ & $0.7 \pm 0.2$ \\
\hline Subparaventricular zone & $42 \pm 3$ & $1.5 \pm 0.2$ \\
\hline Dorsomedial hypothalamic nucleus & $324 \pm 25$ & $11.6 \pm 0.9$ \\
\hline $\begin{array}{l}\text { Ventromedial hypothalamic } \\
\text { nucleus }\end{array}$ & $76 \pm 14$ & $2.7 \pm 0.4$ \\
\hline Lateral hypothalamic area & $501 \pm 138$ & $18.0 \pm 2.7$ \\
\hline Supramammillary area & $58 \pm 17$ & $2.1 \pm 0.8$ \\
\hline Tuberomammillary nucleus & $52 \pm 3$ & $1.9 \pm 0.4$ \\
\hline Perirhinal cortex & $73 \pm 30$ & $2.6 \pm 0.5$ \\
\hline Medial amygdala & $91 \pm 4$ & $3.3 \pm 0.5$ \\
\hline Ventral subiculum & $155 \pm 33$ & $5.6 \pm 0.7$ \\
\hline Raphe nuclei & $82 \pm 23$ & $3.0 \pm 0.4$ \\
\hline Lateral parabrachial nuclei & $137 \pm 24$ & $5.0 \pm 0.9$ \\
\hline Locus coeruleus & $8.3 \pm 2$ & $0.3 \pm 0.1$ \\
\hline $\begin{array}{l}\text { Ventrolateral medulla (A1/C1 } \\
\text { catecholamine groups) }\end{array}$ & $25 \pm 5$ & $0.9 \pm 0.3$ \\
\hline
\end{tabular}

Regions with moderate to high numbers of labeled neurons (comprising $>5 \%$ of total) are highlighted in bold type. Some regions comprising $<1 \%$ of total cells are omitted from the table; hence percentages add up to $<100 \%$.

a more distinct clustering of labeled neurons had been seen. A few labeled neurons were seen in the ventrolateral medulla, in areas overlapping both the $\mathrm{C} 1$ adrenergic and A1 noradrenergic fields (Dahlstrom and Fuxe, 1964; Hökfelt et al., 1974; Tucker et al., 1987). A few retrogradely labeled cells were seen in the ventrolateral periaqueductal gray matter, the locus coeruleus (Fig. 3M), and the nucleus of the solitary tract, with each of these regions contributing $<1 \%$ of ipsilateral retrogradely labeled neurons. Extremely few CTB-labeled neurons were seen in the ventral tegmental area and the central tegmental fields (Fig. $3 K$ ).

\section{Monoaminergic afferents}

Double-label fluorescence immunohistochemistry revealed that most CTB-labeled neurons within the monoaminergic nuclei were also immunoreactive for monoaminergic markers. Most CTB-labeled cells in the TMN were immunoreactive for ADA (Fig. 4A), a marker for histaminergic neurons in the rat (Senba et al., 1985). Double-labeled neurons were found in all TMN subregions, including the ventrolateral, rostral, medial, and caudal groups. Most CTB-labeled neurons in the dorsal raphe, median raphe, and central linear raphe nuclei (Fig. $4 B$ ) were serotonin immunoreactive, but CTB-labeled neurons in the nucleus raphe magnus were never serotonin immunoreactive. Most retrogradely labeled neurons in the locus coeruleus, ventrolateral medulla (both $\mathrm{A} 1$ and $\mathrm{C} 1$ regions), and nucleus of the solitary tract were TH immunoreactive (Fig. $4 C, D$ ), although a minority of CTBlabeled neurons in the nucleus of the solitary tract and the ventrolateral medulla lacked TH immunoreactivity.

In contrast to the other monoamines, extremely few dopaminergic neurons were retrogradely labeled by CTB injections into the
VLPO. The ventral tegmental area showed only occasional CTBlabeled neurons, whereas the substantia nigra never showed CTBlabeled neurons. The hypothalamus contains several THimmunoreactive cell groups (Hokfelt et al., 1984) that are presumably dopaminergic, but hypothalamic $\mathrm{TH}$-immunoreactive neurons were never double labeled with CTB, even in the A11 and A13 dorsal hypothalamic dopamine cell groups where CTB-labeled neurons occasionally intermingled with $\mathrm{TH}$-immunoreactive neurons. A few retrogradely labeled cells just medial to the VLPO injection site were double labeled for $\mathrm{TH}$, but these neurons may have been nonspecifically labeled by dendritic uptake of tracer from the injection site.

\section{Cholinergic, orexin, and melanin concentrating hormone afferents}

Cholinergic inputs to the VLPO appeared rare or nonexistent. Even after large CTB injections into the VLPO, no CTB-labeled cells were immunoreactive for ChAT in any brain area, including the medial septal nucleus, the diagonal band nuclei, the substantia innominata, and brainstem regions such as the laterodorsal tegmental or pedunculopontine tegmental nuclei.

A few CTB-labeled neurons in the LHA were orexin immunoreactive, but fluorescent double labeling showed that orexin neurons accounted for only $4 \%$ ( $n=3$ cases) of all CTB-labeled cells in this area. A larger percentage (21\%;n $n=2$ cases) of CTB-labeled cells in the LHA were immunoreactive for melanin concentrating hormone $(\mathrm{MCH})$. However, most CTB-labeled cells in the LHA were neither orexin nor $\mathrm{MCH}$ immunoreactive and were of undetermined neurochemical identity.

\section{Control injections adjacent to the VLPO}

To control for spread of tracer from VLPO injection sites, we examined CTB injections into areas just adjacent to the VLPO core. Many of these injections produced patterns of retrograde labeling very different from those produced by VLPO injections. Injections into the diagonal band nucleus immediately lateral to the VLPO (cases 1589 and 1782) produced very few labeled neurons in regions strongly labeled by VLPO injections, such as the infralimbic cortex, MnPO, medial preoptic area, SCN, DMH, parabrachial nuclei, and TMN. Instead, diagonal band injections gave rise to retrogradely labeled neurons in the cholinergic laterodorsal tegmental and pedunculopontine tegmental nuclei, areas unlabeled by VLPO injections. CTB injections into the rostral SON (cases 1196 and 1831), which is just ventral and medial to the VLPO, labeled many neurons in the organum vasculosum of the lamina terminalis, the paraventricular nucleus of the hypothalamus, and the parastrial nucleus, areas only sparsely labeled by VLPO core injections that avoided the SON. However, neurons in the $\mathrm{MnPO}$ and subfornical organ were also abundantly labeled by SON injections. CTB injections into the medial preoptic nucleus (Fig. 2, case 2111) labeled a large number of neurons in the ventromedial hypothalamic nucleus, medial amygdaloid nuclei, and ventral premammillary nucleus, areas where VLPO injections produced only low to moderate numbers of labeled neurons. Conversely, medial preoptic injections gave rise to only a few labeled neurons in the MnPO and a small number of labeled neurons in the parabrachial nucleus, areas with many CTB-labeled neurons after VLPO injections.

Two sets of control injections showed a pattern of retrograde labeling very similar to that seen after VLPO core injections. Injections immediately caudal to the VLPO (cases 1738 and 

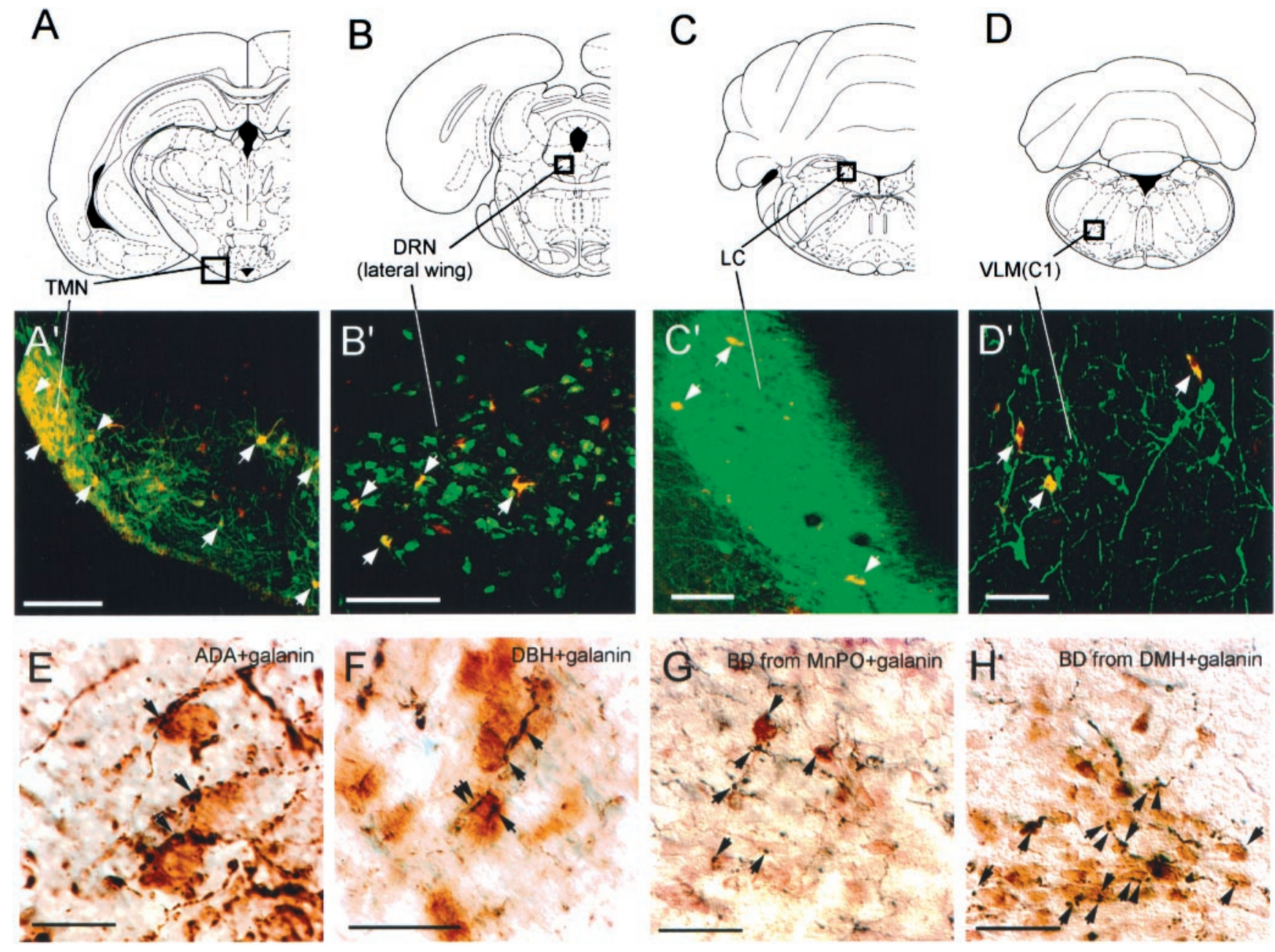

Figure 4. Photomicrographs of double-label immunohistochemistry experiments. $A-D$ (top row) show atlas sections (Paxinos and Watson, 1997) outlining monoaminergic nuclei photographed in panels $A^{\prime}-D^{\prime}$ (middle row). Photographs in $A^{\prime}-D^{\prime}$ are from brains with CTB injections into the VLPO core. Red immunofluorescence indicates CTB-labeled neurons, and green immunofluorescence represents the following: $A^{\prime}$, adenosine-deaminase immunoreactivity (a marker for histaminergic neurons) in the TMN; $B^{\prime}$, serotonin transporter immunoreactivity in the lateral wing of the dorsal raphe; $C^{\prime}, \mathrm{TH}$ immunoreactivity in the locus coeruleus; and $D^{\prime}, \mathrm{TH}$ immunoreactivity in the $\mathrm{C} 1$ adrenergic cells of the ventrolateral medulla (VLM). Double-labeled cells ( yellow) are seen in all four monoaminergic nuclei and are indicated by white arrows. E-H (bottom row) show the V LPO core stained for galanin, shown by brown immunoreactivity. Black immunoreactive fibers are also seen, representing adenosine-deaminase immunoreactivity ( $E$ ) or DBH immunoreactivity $(F)$. Apparent appositions of presumably monoaminergic fibers onto galanin-immunoreactive neurons are seen in both cases and indicated by arrows. $G$ and $H$ show black anterogradely labeled fibers arising from biotinylated dextran injections into the MnPO $(G)$ or DMH $(H)$. Apparent appositions of anterogradely labeled fibers onto galanin-immunoreactive VLPO neurons are indicated by arrows. Scale bars: $A, 200 \mu \mathrm{m} ; B-D$, $100 \mu \mathrm{m} ; E, F, 20 \mu \mathrm{m} ; G, H, 50 \mu \mathrm{m}$.

1880) labeled almost all areas that were labeled by VLPO injections, such as the infralimbic cortex, $\mathrm{MnPO}, \mathrm{DMH}$, lateral septal nucleus, central lateral parabrachial nucleus, and monoaminergic nuclei. Injections immediately dorsal or medial to the VLPO cluster, which presumably involved extended VLPO neurons (Fig. 2, cases 1717 and 1833), also gave rise to a nearly identical pattern of retrogradely labeled cells as seen after VLPO core injections. Hence, neurons in these two regions may share inputs with VLPO core neurons. In particular, the extended VLPO region contains many galaninergic, sleep-active neurons that may be chemically and functionally similar to the VLPO core neurons (Gaus and Saper, 1999). Because these neurons are interspersed with other, nongalaninergic neurons, anterograde tracing is necessary to determine whether the galaninergic extended VLPO neurons share inputs with VLPO core neurons.

\section{Experiment 2: monoaminergic and orexinergic axons in the VLPO}

The VLPO core was densely innervated by fibers immunoreactive for markers of histaminergic, noradrenergic, and serotonergic axons. For each of these three monoamines, roughly 30,000-40,000 varicosities per square millimeter were seen within the VLPO core in 40- $\mu$ m-thick sections (Table 3). Fibers immunoreactive for histamine and $\mathrm{DBH}$, the synthetic enzyme for noradrenaline, were more numerous in the VLPO region than in surrounding regions (Fig. $5 A, B$ ), whereas fibers immunoreactive for 5HTT were equally numerous in the VLPO compared with surrounding regions (Fig. $5 C$ ). Doublelabel immunohistochemistry showed that ADA-immunoreactive fibers and DBH-immunoreactive fibers apposed galanin cell bodies in both the core and extended VLPO (Fig. 4E,F). 


\begin{tabular}{lc}
\hline $\begin{array}{l}\text { Table 3. Densities of varicosities labeled for arousal neurotransmitters } \\
\text { or anterograde tracer in the VLPO core (average of two to three cases } \\
\text { each) }\end{array}$ \\
$\begin{array}{lr} \\
\text { Source of fibers } & \text { Varicosities } \\
\text { Histamine } \text { mm }^{2}\end{array}$ \\
Dopamine- $\boldsymbol{\beta}$-hydroxylase (noradrenaline) & $\mathbf{3 6 , 0 0 0}$ \\
Serotonin transporter & $\mathbf{3 2 , 3 0 0}$ \\
Phenyl-ethanolamine- $N$-methyl transferase & $\mathbf{3 0 , 2 0 0}$ \\
$\quad$ (adrenaline) & \\
Orexin & 5,700 \\
Choline acetyltransferase & 7,700 \\
Infralimbic cortex & 550 \\
Lateral septal nucleus & $\mathbf{1 3 , 4 0 0}$ \\
Median preoptic nucleus & $\mathbf{2 1 , 8 0 0}$ \\
Medial preoptic area & $\mathbf{1 5 , 2 2 5}$ \\
Suprachiasmatic nucleus & 1,850 \\
Subparaventricular zone & 1,050 \\
Dorsomedial hypothalamic nucleus & 1,300 \\
Ventromedial hypothalamic nucleus & $\mathbf{2 5 , 3 0 0}$ \\
Lateral hypothalamic area & \\
Medial amygdala & 1,100 \\
Supramammillary nucleus & 6,350 \\
Lateral parabrachial nucleus & 1,200 \\
\hline & 933 \\
& \\
\hline
\end{tabular}

Moderate to heavy inputs ( $>10,000$ varicosities per square millimeter) are highlighted in bold text. Monoaminergic terminals provide the apparently densest inputs to the VLPO, despite the relatively low numbers of retrogradely labeled monoaminergic neurons.

${ }^{a}$ The lateral hypothalamic area (LHA) is a particularly large brain region in the rat, and our anterograde tracer injections filled only a small fraction of this area; hence, varicosities arising from LHA injections may underestimate the intensity of this afferent to the VLPO.

5HTT-immunoreactive fibers were only visible after silver enhancement, which is incompatible with black-brown doublelabel immunohistochemistry. Hence, we were unable simultaneously to visualize 5HTT fibers and galanin neurons, but the high density of 5HTT fibers throughout the preoptic area suggests substantial opportunity for interaction with VLPO galaninergic neurons.

DBH immunostaining cannot distinguish between noradrenergic and adrenergic inputs to the VLPO. Hence, we examined the distribution of the enzyme phenylethanolamine- $N$-methyltransferase (PNMT), which converts noradrenaline to adrenaline and is expressed in adrenergic but not noradrenergic neurons and fibers. PNMT-immunoreactive axons and boutons innervated the VLPO in a pattern similar to that of DBH fibers, but with a much lower density (Fig. 5D, Table 3). Hence, most DBH fibers in the VLPO are probably noradrenergic, whereas a minority are probably adrenergic.

Low to moderate densities of orexin fibers and boutons in the VLPO core were seen (Fig. 5E, Table 3 ). The VLPO contained only sparse ChAT immunoreactive fibers (Fig. $5 F$, Table 3 ) and virtually no DAT immunoreactive fibers, although nearby brain areas showed dense innervation by DAT- or ChATimmunoreactive fibers. Although DAT immunoreactivity may underestimate the density of dopaminergic input to the VLPO because DAT levels are relatively low in ventral tegmental and hypothalamic dopaminergic neurons (Freed et al., 1995), our retrograde studies found very few CTB-labeled dopaminergic neurons in these regions.

\section{Experiment 3: anterograde tracing}

We injected the anterograde tracers BD, PHAL, or WGA-HRP into most areas labeled by CTB injections into the VLPO, including the infralimbic cortex, lateral septal nucleus, MnPO, medial preoptic area, DMH, SCN, subparaventricular zone, ventromedial hypothalamic nucleus, LHA, supramammillary nucleus, medial amygdala, central lateral parabrachial nucleus, and nucleus of the solitary tract. We did not inject anterograde tracers into the monoaminergic nuclei because immunostaining for monoaminergic markers already demonstrated the presence of monoaminergic fibers in the VLPO.

Many fibers and boutons apposed to VLPO core neurons were seen after anterograde injections into seven areas: the infralimbic cortex, lateral septal nucleus, MnPO, DMH, lateral hypothalamic area, ventral subiculum, and central lateral parabrachial nucleus (Fig. 6A-F). Because densities of fibers and boutons varied greatly with the size of the anterograde tracer injection, we only quantified varicosities arising from tracer injections that primarily filled the nuclei of interest. These counts showed varicosity densities ranging from 6000 to 25000 per square millimeter for these seven regions, which is roughly comparable to the monoaminergic varicosity densities. We did not quantify anterograde labeling arising from the ventral subiculum, because this area was injected with WGA-HRP, a tracer that does not label the fine structure of axons. Anterograde tracer injections just outside of these seven areas gave rise to very few anterogradely labeled fibers in the VLPO, suggesting that afferent pathways arose specifically from within these cell groups and not from fibers of passage originating elsewhere. Notably, these seven regions in which anterograde tracer injections produced the most boutons in the VLPO core were also the seven regions with the most retrogradely labeled neurons (Tables 2, 3), suggesting good agreement between anterograde and retrograde tracing experiments.

Anterogradely labeled fibers and varicosities were particularly intense after injections into the lateral septal nucleus, the MnPO, and the DMH. To further confirm some of these inputs to the VLPO, we performed double labeling for $\mathrm{BD}$ and galanin and found that axons and boutons arising from the $\mathrm{MnPO}$ and $\mathrm{DMH}$ apposed galanin-immunoreactive neurons in both the core and extended VLPO (Fig. 4G,H). Because colchicine treatment is required to visualize galanin immunoreactivity in cell bodies, other afferents could not be confirmed in this manner.

Anterograde injections into the LHA produced moderate numbers of varicosities in the VLPO core, regardless of whether the LHA injections were dorsal, lateral, or ventral to the fornix. Because the LHA is a large region, each of our anterograde injections filled only a small fraction of this area. Hence, the LHA projection to the VLPO is likely to be denser than indicated by any individual injection.

Only a few fibers and boutons were seen in the VLPO core after BD injections into six areas: the medial preoptic nucleus, suprachiasmatic nucleus, subparaventricular zone, ventromedial hypothalamic nucleus, medial amygdala, and supramammillary nucleus (Table 3). In these cases, many anterogradely labeled axons and boutons terminated just outside the VLPO core. Injections into a seventh region, the nucleus of the solitary tract, also produced very few fibers in the VLPO core, but with almost no fibers terminating just outside the VLPO core. In these regions, only low to moderate numbers of retrogradely labeled neurons had been seen after CTB injections into the VLPO, again showing rough agreement between anterograde and retrograde tracing experiments. 

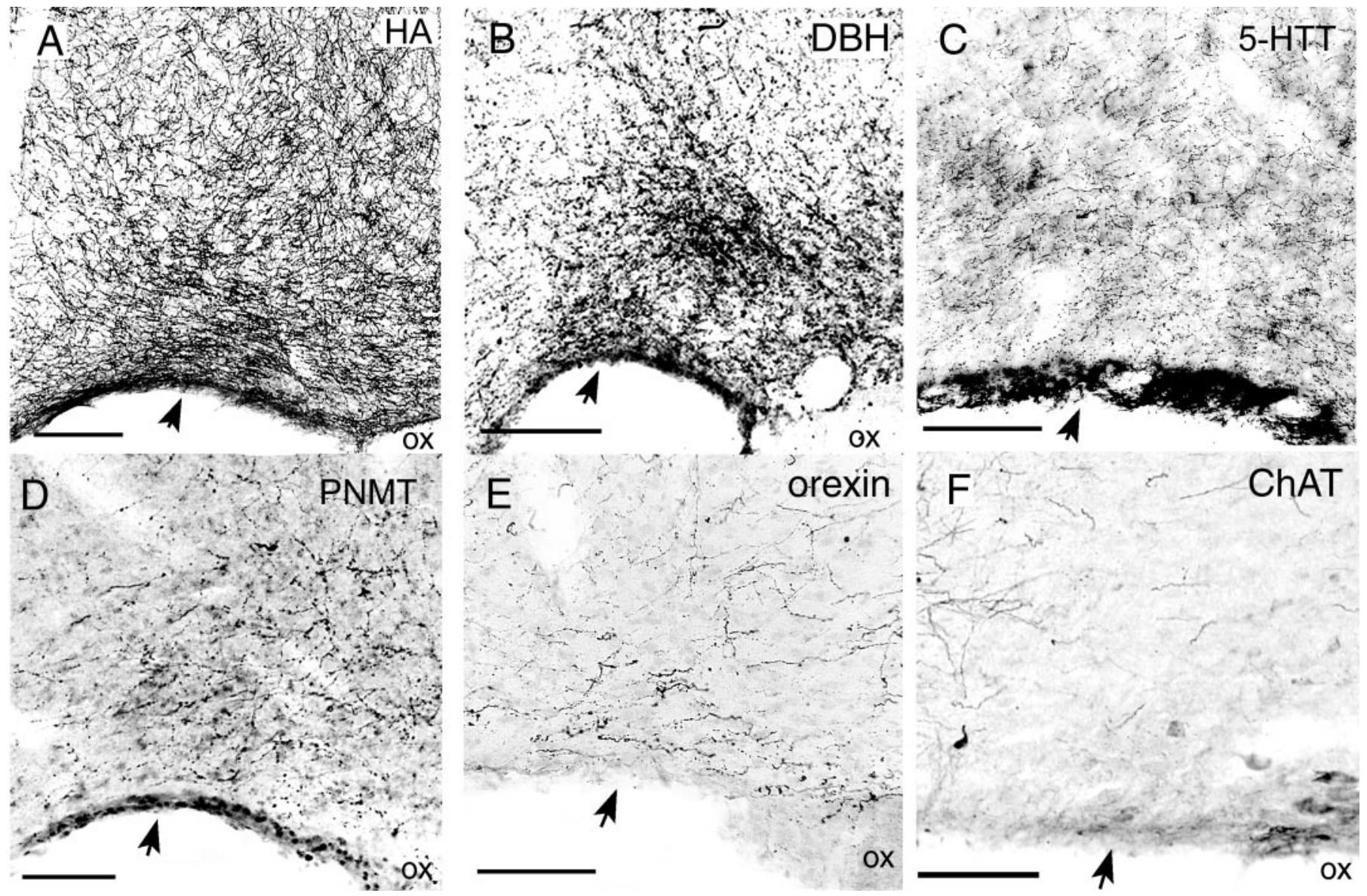

Figure 5. Photomicrographs of VLPO area showing fibers immunoreactive for histamine $(A, H A)$; DBH $(B)$, the synthetic enzyme for noradrenaline; 5HTT $(C)$; PNMT $(D)$, the synthetic enzyme for adrenaline; orexin $(E)$, and ChaT $(F)$. The VLPO core is indicated by arrows. Histamine fibers are most numerous in the VLPO core, whereas DBH fibers are most numerous in the extended VLPO, just dorsomedial to the VLPO core. Orexin fiber innervation of the VLPO is relatively modest, whereas ChAT fibers are extremely sparse in the VLPO, although ChAT fibers are abundant in the adjacent diagonal band. Scale bars: $A, B, 250 \mu \mathrm{m} ; C-F, 100 \mu \mathrm{m}$.

Most $\mathrm{BD}$ injections gave rise to a few retrogradely labeled neurons, consistent with previous reports that BD is weakly transported in the retrograde direction (Brandt and Apkarian, 1992). Hence, anterogradely labeled fibers in the VLPO could have arisen if these retrogradely labeled neurons sent branched axons to both the VLPO and the injection site. However, BDlabeled cells were sparsely distributed and often faintly labeled, and most did not occur in regions projecting to the VLPO. In cases in which BD-labeled cells occurred in areas projecting to the VLPO, we verified our results with another anterograde tracer, PHAL, which is not transported retrogradely. After PHAL injections into the infralimbic cortex, $\mathrm{DMH}$, and nucleus of the solitary tract, PHAL-labeled fibers innervated the VLPO core in a pattern very similar to that seen after BD injections.

\section{DISCUSSION}

Retrograde tracing, anterograde tracing, and double-label immunohistochemistry showed that the VLPO receives intense input from several monoaminergic systems, as well as inputs from specific hypothalamic, limbic, and autonomic regions (Fig. 7). The VLPO receives moderate orexin and $\mathrm{MCH}$ inputs but little or no dopaminergic or cholinergic inputs. In conjunction with previous findings, our data also suggest that sleep-active VLPO neurons and wake-active monoaminergic neurons innervate each other.

\section{Technical considerations}

The VLPO core is an extremely small area, and even our smallest injections of CTB into the VLPO core diffused slightly into adjacent regions, requiring the use of anterograde tracing to determine whether an area projected to the VLPO core. The anterograde tracer BD may exhibit weak retrograde transport and may be taken up by fibers of passage (Brandt and Apkarian, 1992). However, BD-labeled retrogradely labeled cells were infrequent, faintly labeled, and usually not found in regions projecting to the VLPO. Control injections of BD into areas near those projecting to the VLPO did not produce labeled fibers in the VLPO, suggesting that fibers of passage did not contribute substantially to anterograde labeling in the VLPO. Finally, the anterograde tracer PHAL, which is not retrogradely transported or taken up by fibers of passage, gave results similar to BD injections into the DMH and parabrachial nuclei. CTB is not thought to be taken up by undamaged fibers of passage (Luppi et al., 1990).

Counts of anterogradely labeled varicosities and retrogradely labeled cells were highly dependent on the size of the injections. To reduce this variability, we calculated retrograde labeling in each region as a percentage of total ipsilateral cells and quantified anterograde labeling only after injections that primarily filled the 

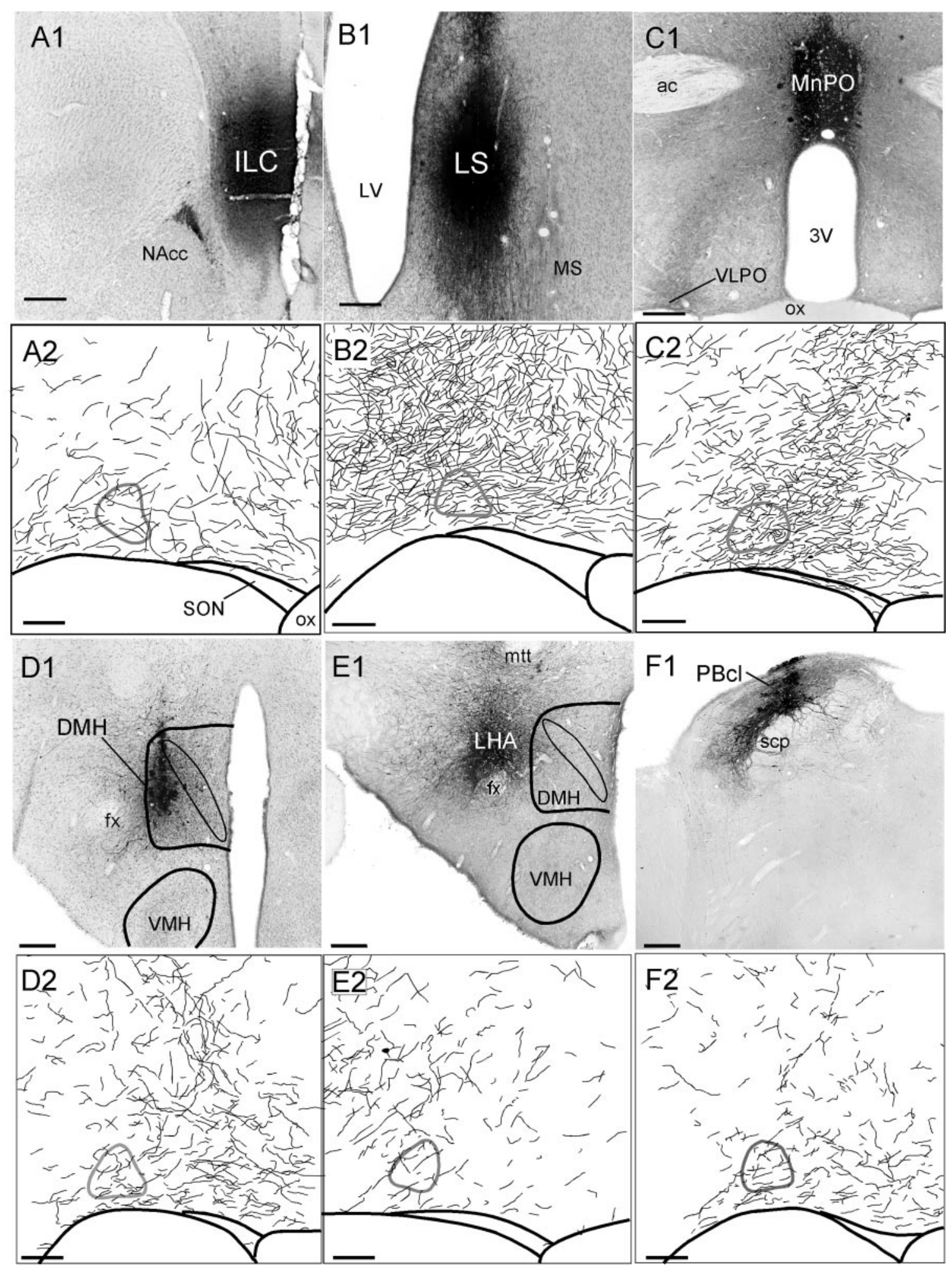

Figure 6. Anterograde tracer injections into infralimbic cortex $(A)$, lateral septal nucleus $(B)$, median preoptic nucleus $(C)$, dorsomedial hypothalamic nucleus $(D)$, lateral hypothalamic area $(E)$, lateral parabrachial nucleus $(F) . A 1-F 1$, Photomicrographs of BD injection sites. Scale bars, $250 \mu \mathrm{m}$. $A 2-F 2$, Camera lucida drawings of anterogradely labeled fibers in the VLPO. Scale bars, $100 \mu \mathrm{m}$. The VLPO core, identified by Nissl counterstain, is outlined in gray.

nucleus of interest. However, varicosity densities were still somewhat variable and should be interpreted only as a rough estimate of the size of a projection.

Quantitative results from our retrograde and anterograde transport experiments generally corroborated each other, except for the monoaminergic nuclei. The monoaminergic nuclei had only modest numbers of CTB-labeled neurons in our retrograde tracing experiment, but we found very high densities of histamin- 


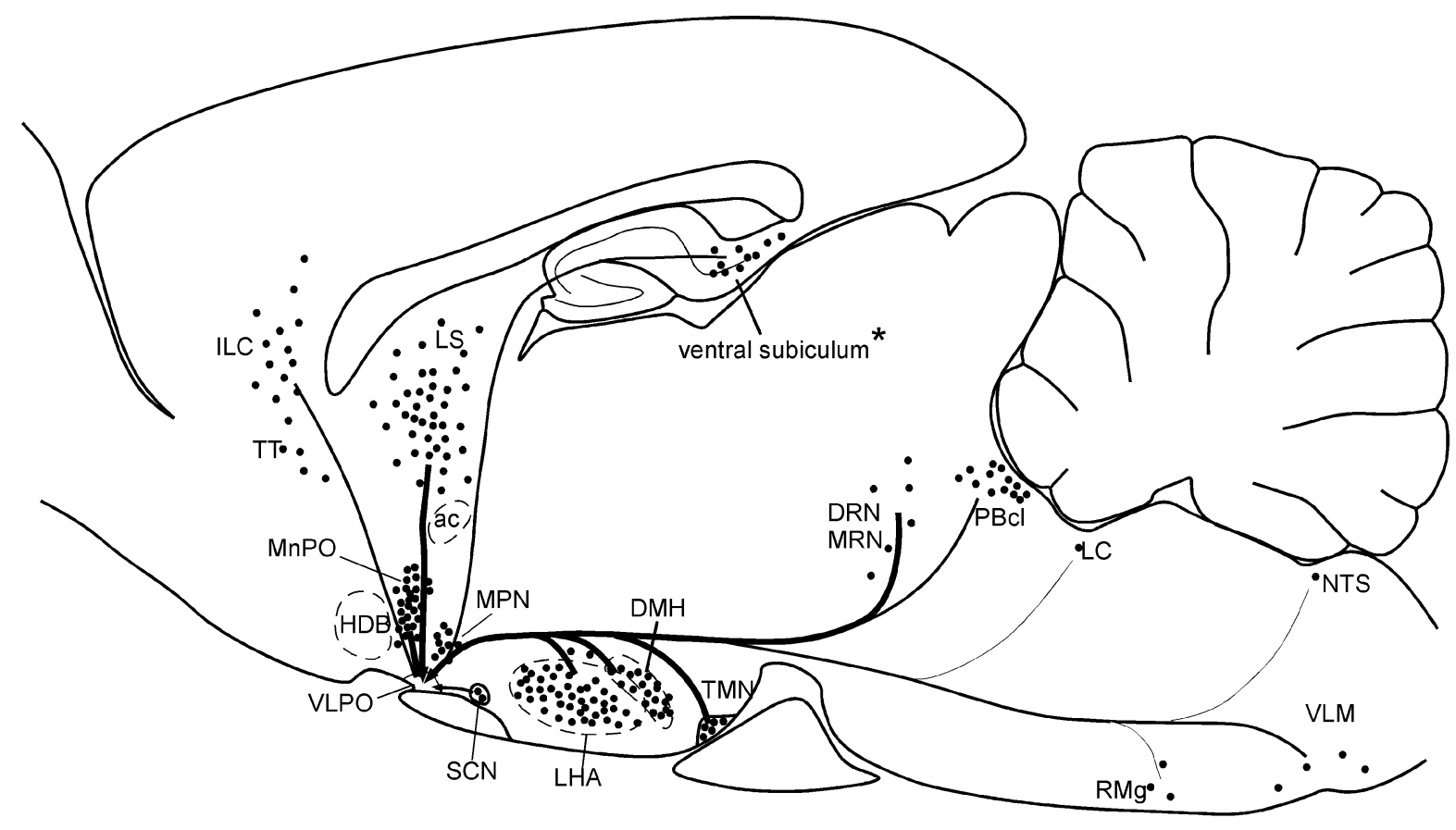

Figure 7. Sagittal summary diagram of the afferents to the VLPO. Major regions projecting to the VLPO are indicated by dots, with one dot indicating roughly 10 retrogradely labeled neurons (counted in every fifth section, average of 3 cases). Line thickness roughly indicates intensity of varicosity density in VLPO core, which does not always correlate with density of retrograde labeling. *Because the ventral subiculum does not appear in the sagittal level shown, retrogradely labeled neurons in the ventral subiculum are depicted more dorsally than they appear in the brain.

ergic, serotoninergic, and noradrenergic varicosities in the VLPO core. Although this apparent discrepancy could be caused by variations in immunostaining versus retrograde transport efficiency, another explanation is that monoaminergic axons are known to be heavily branched (Saper, 1985). Our findings are consistent with this possibility that relatively few monoaminergic neurons may produce large numbers of axons and varicosities.

Anterograde injections into the LHA also appeared to give rise to fewer labeled axons in the VLPO than expected on the basis of retrograde injections. However, the explanation here may be that the LHA is such a large area that no single anterograde injection filled more than a small fraction of this region. Hence we were able to label only a small part of the LHA projection to the VLPO with any single anterograde tracer injection.

\section{Comparison with previous studies}

The VLPO has only recently been identified as a distinct nucleus using physiological, anatomical, and neurochemical criteria. Although many studies have examined inputs to the preoptic area, only a few distinguished the VLPO from surrounding cell groups, most notably works by Swanson and colleagues (Canteras et al., 1994; Thompson et al., 1996; Swanson, 1998) who used the term anteroventral preoptic nucleus to describe the VLPO core. The current findings are consistent with previous studies that have found projections to the lateral preoptic area from the lateral parabrachial nucleus (Saper and Loewy, 1980; Bester et al., 1997), the DMH (Thompson et al., 1996), and the infralimbic cortex (Hurley et al., 1991). The current results are also consistent with previous findings of a strong histaminergic innervation of the lateral preoptic area in cats (Lin et al., 1994).

\section{Functional implications}

In conjunction with previous findings, the current results suggest that the VLPO and monoaminergic systems strongly innervate each other and may also be mutually inhibitory. VLPO neurons project to the monoaminergic nuclei and contain the inhibitory transmitter GABA (Sherin et al., 1998) and also contain galanin, which inhibits neurons in the TMN (Schonrock et al., 1991), raphe nuclei (Xu et al., 1998), and locus coeruleus (Sevcik et al., 1993). Conversely, noradrenaline and serotonin inhibit GABAergic neurons in the VLPO area (Gallopin et al., 2000). However, it is unclear whether these GABAergic neurons are indeed VLPO neurons, because many cell groups surrounding the VLPO also contain GABA (Sherin et al., 1998).

Although we found that histaminergic fibers densely innervate the VLPO, histamine has not been shown to influence VLPO firing rates (Gallopin et al., 2000). However, TMN neurons, which are the only neuronal source of histamine, also contain galanin and GABA (Airaksinen et al., 1992), which could influence VLPO activity even if histamine does not. TMN fibers could also presynaptically modulate other inputs to the VLPO, an effect that might not be detected in the slice preparations used by Gallopin and colleagues (2000).

If the VLPO and arousal systems are reciprocally inhibitory, the wake-active and sleep-active halves of this system would each disinhibit their own firing. Hence, activity of either sleep or arousal systems would be self-reinforcing. Such a mutually inhibitory network could exhibit bistable firing patterns (Horowitz and Hill, 1989; Kirillov et al., 1993), which would resist changes between sleep and wake states, whereas the positive feedback loop in this circuit would ensure that behavioral state transitions occur rapidly once initiated. Such a bistable sleep-wake "switch" would be behaviorally advantageous, prolonging both sleep and wake bouts while minimizing the duration of transitions between sleep and wake states.

Although histaminergic, noradrenergic, and serotonergic fibers densely innervate the VLPO, the current work shows almost no 
dopaminergic input to the VLPO. Interestingly, the firing rates of dopaminergic neurons in the substantia nigra may not be reduced during sleep in cats (Trulson, 1985), in contrast to the behavior of other monoaminergic systems with firing rates that diminish during sleep (McGinty and Harper, 1976; Hobson et al., 1983; Vanni-Mercier et al., 1984). Hence, the anatomic and functional relationship of the dopaminergic system to sleep-wake control may be substantially different from other monoaminergic systems.

We did not find substantial cholinergic projections to the VLPO from either the basal forebrain or brainstem using retrograde tracing combined with ChAT immunostaining. This result is notable because acetylcholine inhibits GABAergic neurons in the VLPO area in vitro (Gallopin et al., 2000). Further research is necessary to explain whether and how the VLPO may respond to acetylcholine in the absence of substantial cholinergic innervation.

The temporal distribution of sleep is strongly influenced by the endogenous circadian pacemaker in the SCN (Eastman et al., 1984; Dijk and Czeisler, 1995). However, the VLPO receives only sparse inputs from the SCN (Gaus and Saper, 1998; Novak and Nunez, 2000; Sun et al., 2000). The current study confirms these findings and also finds that the VLPO receives only sparse input from the SPZ, a major target of SCN efferents. However, the DMH receives moderate inputs from the SCN and SPZ (Watts et al., 1987; Thompson et al., 1996), and we saw a dense projection from the DMH to the VLPO. We recently found that DMH lesions markedly decrease the circadian amplitude of sleep, although they leave body temperature rhythms intact (Chou et al., 2000). Hence, circadian signals may influence the circadian timing of sleep via a neuronal relay in the $\mathrm{DMH}$; this topic will be the focus of a later communication (Gaus et al., 2000).

Other afferents to the VLPO may also have important effects on sleep. The lateral parabrachial subnuclei respond to painful stimuli (Lanteri-Minet et al., 1994; Bester et al., 2000), and the strong projection from this area to the VLPO may play a role in the alterations of sleep induced by pain. The VLPO receives inputs from the infralimbic cortex and nucleus of the solitary tract, areas implicated in the regulation of cardiovascular, respiratory, and other autonomic functions (Cechetto and Saper, 1987; Machado et al., 1997). Hence, changes in autonomic function could influence sleep via these pathways. The MnPO has been implicated in fluid homeostasis and body temperature (Wilkin et al., 1989) and shows increased Fos expression during sleep, particularly in a warm environment (Gong et al., 2000), suggesting possible interactions between ambient temperature, homeostatic states, and sleep. The VLPO receives strong inputs from the lateral septal nucleus and ventral subiculum, which are strongly innervated by the hippocampus, suggesting possible interactions between hippocampal functions and sleep. Finally, the largest source of inputs to the VLPO may be the lateral hypothalamic area. This area serves many functions related to feeding and arousal and may play an important role in sleep-wake regulation via its reciprocal connectivity with the VLPO.

Further anatomic and physiologic studies are needed to determine the functions of the afferents to the VLPO. These pathways may help explain many important interactions between sleep and circadian, autonomic, sensory, and limbic functions.

\section{REFERENCES}

Airaksinen MS, Alanen S, Szabat E, Visser TJ, Panula P (1992) Multiple neurotransmitters in the tuberomammillary nucleus: comparison of rat, mouse, and guinea pig. J Comp Neurol 323:103-116.

Alfoldi P, Franken P, Tobler I, Borbely AA (1991) Short light-dark cycles influence sleep stages and EEG power spectra in the rat. Behav Brain Res [Erratum (1991) 44:233]43:125-131.

Bester H, Besson JM, Bernard JF (1997) Organization of efferent projections from the parabrachial area to the hypothalamus: a Phaseolus vulgaris-leucoagglutinin study in the rat. J Comp Neurol 383:245-281.

Bester H, Beggs S, Woolf CJ (2000) Changes in tactile stimuli-induced behavior and c-Fos expression in the superficial dorsal horn and in parabrachial nuclei after sciatic nerve crush. J Comp Neurol 428:45-61.

Brandt HM, Apkarian AV (1992) Biotin-dextran: a sensitive anterograde tracer for neuroanatomic studies in rat and monkey. J Neurosci Methods 45:35-40.

Canteras NS, Simerly RB, Swanson LW (1994) Organization of projections from the ventromedial nucleus of the hypothalamus: a Phaseolus vulgaris-leucoagglutinin study in the rat. J Comp Neurol 348:41-79.

Cechetto DF, Saper CB (1987) Role of the cerebral cortex in autonomic function. In: Central regulation of autonomic functions (Loewy A, Spyer KM, eds), pp 208-223. New York: Oxford UP.

Chou T, Lu J, Scammell T, Shiromani P, Saper C (2000) Excitotoxic lesions of the dorsomedial hypothalamic nucleus markedly attenuate circadian rhythms of sleep and body temperature. Soc Neurosci Abstr 26:469.9.

Dahlstrom A, Fuxe K (1964) Evidence of the existence of monoamine containing neurons in the central nervous system. I. Demonstration of monoamines in the cell bodies of brain stem neurons. Acta Physiol Scand 62:1-55.

Dijk DJ, Czeisler CA (1995) Contribution of the circadian pacemaker and the sleep homeostat to sleep propensity, sleep structure, electroencephalographic slow waves, and sleep spindle activity in humans. J Neurosci 15:3526-3538.

Eastman CI, Mistlberger RE, Rechtschaffen A (1984) Suprachiasmatic nuclei lesions eliminate circadian temperature and sleep rhythms in the rat. Physiol Behav 32:357-368.

Elmquist JK, Saper CB (1996) Activation of neurons projecting to the paraventricular hypothalamic nucleus by intravenous lipopolysaccharide. J Comp Neurol 374:315-331.

Freed C, Revay R, Vaughan RA, Kriek E, Grant S, Uhl GR, Kuhar MJ (1995) Dopamine transporter immunoreactivity in rat brain. J Comp Neurol 359:340-349.

Gallopin T, Fort P, Eggermann E, Cauli B, Luppi PH, Rossier J, Audinat E, Muhlethaler M, Serafin M (2000) Identification of sleep-promoting neurons in vitro. Nature 404:992-995.

Gaus SE, Saper CB (1998) Efferent connections from the suprachiasmatic nucleus to the ventrolateral preoptic nucleus in the rat. Soc Neurosci Abstr 24:1920.

Gaus SE, Saper CB (1999) Sleep-active neurons in the ventrolateral preoptic nucleus (VLPO) are galaninergic. Soc Neurosci Abstr 25:625

Gaus SE, Chou TC, Saper CB (2000) Circadian control of sleep: disynaptic connections from the suprachiasmatic nucleus to the ventrolateral preoptic nucleus. Soc Neurosci Abstr 26:2024.

Gong H, Szymusiak R, King J, Steininger T, McGinty D (2000) Sleeprelated c-Fos protein expression in the preoptic hypothalamus: effects of ambient warming. Am J Physiol Regul Integr Comp Physiol 279:R2079-2088.

Hobson JA, McCarley RW, Nelson JP (1983) Location and spike-train characteristics of cells in anterodorsal pons having selective decreases in firing rate during desynchronized sleep. J Neurophysiol 50:770-783.

Hökfelt T, Fuxe K, Goldstein M, Johansson O (1974) Immunohistochemical evidence for the existence of adrenaline neurons in the rat brain. Brain Res 66:235-251.

Hokfelt T, Martensson R, Bjorklund A, Kleinau S, Goldstein M (1984) Distributional maps of tyrosine-hydroxylase-immunoreactive neurons in the rat brain. In: Handbook of chemical neuroanatomy, Vol 2, Classical transmitters in the CNS, Part 1 (Bjorklund A, Hokfelt T, eds) pp 277-379. Amsterdam: Elsevier.

Horowitz P, Hill W (1989) The Art of electronics. Cambridge: Cambridge UP.

Hurley KM, Herbert H, Moga MM, Saper CB (1991) Efferent projections of the infralimbic cortex of the rat. J Comp Neurol 308:249-276.

Kirillov AB, Myre CD, Woodward DJ (1993) Bistability, switches and working memory in a two-neuron inhibitory-feedback model. Biol Cybern 68:441-449.

Kitt C (1988) Immunocytochemical visualization of cholinergic fibers in monkey neocortex: enhanced visualization using silver nitrate. Soc Neurosci Abstr 14:631.

Lanteri-Minet M, Weil-Fugazza J, de Pommery J, Menetrey D (1994) Hindbrain structures involved in pain processing as revealed by the expression of c-Fos and other immediate early gene proteins. Neuroscience 58:287-298.

Lin JS, Sakai K, Jouvet M (1994) Hypothalamo-preoptic histaminergic projections in sleep-wake control in the cat. Eur J Neurosci 6:618-625.

Lu J, Shiromani P, Saper CB (1999) Retinal input to the sleep-active ventrolateral preoptic nucleus in the rat. Neuroscience 93:209-214.

Lu J, Greco MA, Shiromani P, Saper CB (2000a) Effect of lesions of the ventrolateral preoptic nucleus on NREM and REM sleep. J Neurosci 20:3830-3842. 
Lu J, Xu M, Gaus SE, Shiromani PJ, Saper CB (2000b) The extended ventrolateral preoptic nucleus regulates REM sleep. Soc Neurosci Abstr 26:2024.

Luppi PH, Fort P, Jouvet M (1990) Iontophoretic application of unconjugated cholera toxin B subunit (CTb) combined with immunohistochemistry of neurochemical substances: a method for transmitter identification of retrogradely labeled neurons. Brain Res 534:209-224.

Machado BH, Mauad H, Chianca Junior DA, Haibara AS, Colombari E (1997) Autonomic processing of the cardiovascular reflexes in the nucleus tractus solitarii. Braz J Med Biol Res 30:533-543.

Matsumura H, Nakajima T, Osaka T, Satoh S, Kawase K, Kubo E, Kantha SS, Kasahara K, Hayaishi O (1994) Prostaglandin D2-sensitive, sleeppromoting zone defined in the ventral surface of the rostral basal forebrain. Proc Natl Acad Sci USA 91:11998-12002.

McGinty DJ, Harper RM (1976) Dorsal raphe neurons: depression of firing during sleep in cats. Brain Res 101:569-755.

McGinty D, Szymusiak R, Thomson D (1994) Preoptic/anterior hypothalamic warming increases EEG delta frequency activity within nonrapid eye movement sleep. Brain Res 667:273-277.

Mesulam MM (1978) Tetramethyl benzidine for horseradish peroxidase neurohistochemistry: a non-carcinogenic blue reaction product with superior sensitivity for visualizing neural afferents and efferents. J Histochem Cytochem 26:106-117.

Novak CM, Nunez AA (2000) A sparse projection from the suprachiasmatic nucleus to the sleep active ventrolateral preoptic area in the rat. NeuroReport 11:93-96.

Paxinos G, Watson C (1997) The rat brain in stereotaxic coordinates. San Diego: Academic.

Roehrs T, Zorick F, Roth T (1994) Transient and short-term insomnia. In: Principles and practice of sleep medicine (Kryger MH, Roth TR, Dement WC, eds), pp 000-000. Philadelphia: W. B. Saunders.

Sakaguchi S, Glotzbach SF, Heller HC (1979) Influence of hypothalamic and ambient temperatures on sleep in kangaroo rats. Am J Physiol 237:R80-88.

Saper CB (1985) Diffuse cortical projection systems: anatomical organization and role in cortical function In: Handbook of physiology-the nervous system. (Plum F, ed), pp 169-210. Bethesda, MD: Am Physiol Soc.

Saper CB, Loewy AD (1980) Efferent connections of the parabrachial nucleus in the rat. Brain Res 197:291-317.

Scammell T, Gerashchenko D, Urade Y, Onoe H, Saper C, Hayaishi O (1998a) Activation of ventrolateral preoptic neurons by the somnogen prostaglandin D2. Proc Natl Acad Sci USA 95:7754-7759.

Scammell TE, Griffin JD, Elmquist JK, Saper CB (1998b) Microinjection of a cyclooxygenase inhibitor into the anteroventral preoptic region attenuates LPS fever. Am J Physiol 274:R783-789.

Scammell TS, Gerashchenko D, Mochizuki T, McCarthy M, Estabrooke I, Sears C, Saper CB, Urade Y, Hayaishi O (2001) An adenosine A2a agonist increases sleep and induces Fos in ventrolateral preoptic neurons. Neuroscience 107:653-663.

Schonrock B, Busselberg D, Haas H (1991) Properties of tuberomam- millary histamine neurones and their response to galanin. Agents Actions 33:135-137.

Senba E, Daddona PE, Watanabe T, Wu JY, Nagy JI (1985) Coexistence of adenosine deaminase, histidine decarboxylase, and glutamate decarboxylase in hypothalamic neurons of the rat. $\mathrm{J}$ Neurosci 5:3393-3402.

Sevcik J, Finta EP, Illes P (1993) Galanin receptors inhibit the spontaneous firing of locus coeruleus neurones and interact with mu-opioid receptors. Eur J Pharmacol 230:223-230.

Sherin JE, Shiromani PJ, McCarley RW, Saper CB (1996) Activation of ventrolateral preoptic neurons during sleep. Science 271:216-219.

Sherin JE, Elmquist JK, Torrealba F, Saper CB (1998) Innervation of histaminergic tuberomammillary neurons by GABAergic and galaninergic neurons in the ventrolateral preoptic nucleus of the rat. J Neurosci 18:4705-4721.

Steininger TL, Gong H, McGinty D, Szymusiak R (2001) Subregional organization of preoptic area/anterior hypothalamic projections to arousal-related monoaminergic cell groups. J Comp Neurol 429:638-653.

Sun X, Rusak B, Semba K (2000) Electrophysiology, pharmacology of projections from the suprachiasmatic nucleus to the ventromedial preoptic area in rat. Neuroscience 98:715-728.

Swanson LW (1998) Brain maps: structure of the rat brain. Amsterdam: Elsevier.

Szymusiak R, Alam N, Steininger TL, McGinty D (1998) Sleep-waking discharge patterns of ventrolateral preoptic/anterior hypothalamic neurons in rats. Brain Res 803:178-188.

Thompson RH, Canteras NS, Swanson LW (1996) Organization of projections from the dorsomedial nucleus of the hypothalamus: a PHA-L study in the rat. J Comp Neurol 376:143-173.

Trulson ME (1985) Activity of dopamine-containing substantia nigra neurons in freely moving cats. Neurosci Biobehav Rev 9:283-297.

Tucker DC, Saper CB, Ruggiero DA, Reis DJ (1987) Organization of central adrenergic pathways: I. Relationships of ventrolateral medullary projections to the hypothalamus and spinal cord. J Comp Neurol 259:591-603.

Vanni-Mercier G, Sakai K, Jouvet M (1984) Specific neurons for wakefulness in the posterior hypothalamus in the cat. C R Acad Sci III 298:195-200.

Watts AG, Swanson LW, Sanchez-Watts G (1987) Efferent projections of the suprachiasmatic nucleus: I. Studies using anterograde transport of Phaseolus vulgaris leucoagglutinin in the rat. J Comp Neurol 258:204-229.

Wilkin LD, Mitchell LD, Ganten D, Johnson AK (1989) The supraoptic nucleus: afferents from areas involved in control of body fluid homeostasis. Neuroscience 28:573-584.

Xu ZQ, Zhang X, Pieribone VA, Grillner S, Hokfelt T (1998) Galanin5-hydroxytryptamine interactions: electrophysiological, immunohistochemical and in situ hybridization studies on rat dorsal raphe neurons with a note on galanin R1 and R2 receptors. Neuroscience 87:79-94. 